\title{
AN ALMA DISK MASS FOR THE CANDIDATE PROTOPLANETARY COMPANION TO FW TAU
}

\author{
Adam L. Kraus ${ }^{1}$, Sean M. Andrews ${ }^{2}$, Brendan P. Bowler ${ }^{3,10}$, Gregory Herczeg $^{4}$, Michael J. Ireland ${ }^{5}$, \\ Michael C. LiU ${ }^{6}$, Stanimir Metchev $^{7}$, and Kelle L. Cruz ${ }^{8,9}$ \\ ${ }^{1}$ Department of Astronomy, The University of Texas at Austin, Austin, TX 78712, USA \\ ${ }^{2}$ Harvard-Smithsonian Center for Astrophysics, 60 Garden Street, Cambridge, MA 02138, USA \\ ${ }^{3}$ California Institute of Technology, Division of Geological and Planetary Sciences, 1200 East California Boulevard, Pasadena, CA 91101, USA \\ ${ }^{4}$ Kavli Institute for Astronomy and Astrophysics, Peking University, Yi He Yuan Lu 5, Haidian Qu, Beijing 100871, China \\ ${ }^{5}$ Research School of Astronomy \& Astrophysics, Australian National University, Canberra ACT 2611, Australia \\ ${ }^{6}$ Institute for Astronomy, University of Hawai'i, 2680 Woodlawn Drive, Honolulu, HI 96822, USA \\ ${ }^{7}$ Department of Physics and Astronomy, The University of Western Ontario, London, ON N6A 3K7, Canada \\ ${ }^{8}$ Department of Physics and Astronomy, Hunter College, City University of New York, New York, NY 10065, USA \\ ${ }^{9}$ Astrophysics Department, American Museum of Natural History, New York, NY 10024, USA \\ Received 2014 September 9; accepted 2014 December 4; published 2014 December 19
}

\begin{abstract}
We present ALMA observations of the FW Tau system, a close binary pair of M5 stars with a wide-orbit (300 AU projected separation) substellar companion. The companion is extremely faint and red in the optical and near-infrared, but boasts a weak far-infrared excess and optical/near-infrared emission lines indicative of a primordial accretion disk of gas and dust. The component-resolved $1.3 \mathrm{~mm}$ continuum emission is found to be associated only with the companion, with a flux $(1.78 \pm 0.03 \mathrm{mJy})$ that indicates a dust mass of $1-2 M_{\oplus}$. While this mass reservoir is insufficient to form a giant planet, it is more than sufficient to produce an analog of the Kepler-42 exoplanetary system or the Galilean satellites. The mass and geometry of the disk-bearing FW Tau companion remains unclear. Near-infrared spectroscopy shows deep water bands that indicate a spectral type later than M5, but substantial veiling prevents a more accurate determination of the effective temperature (and hence mass). Both a disk-bearing "planetary-mass" companion seen in direct light or a brown dwarf tertiary viewed in light scattered by an edge-on disk or envelope remain possibilities.
\end{abstract}

Key words: planets and satellites: formation - planets and satellites: general - protoplanetary disks

\section{INTRODUCTION}

Over the past decade, direct imaging surveys have discovered a small but significant number of faint, apparently planetarymass $\left(\lesssim 20 M_{\text {Jup }}\right)$ companions that orbit their primary star hosts $\left(M \sim 0.2-1.5 M_{\odot}\right)$ at ultrawide separations $(\gtrsim 100 \mathrm{AU}$, extending to thousands of AU; Neuhäuser et al. 2005; Lafrenière et al. 2008; Schmidt et al. 2008; Ireland et al. 2011; Bailey et al. 2014; Kraus et al. 2014). These planetary mass companions (PMCs) represent intriguing analogs to the recent discoveries of smaller separation planets, like HR 8799 bcde (Marois et al. 2008), Beta Pic b (Lagrange et al. 2009), HD 95086 b (Rameau et al. 2013), GJ 504 b (Kuzuhara et al. 2013), and LkCa15 b (Kraus \& Ireland 2012). The large orbital separations of the PMCs are markedly different from the planets in our own solar system and the vast population of exoplanets detected with the radial velocity and transit methods, so it is not clear whether PMCs formed via similar processes. Planets at orbital radii of $\lesssim 100$ AU can feasibly be formed via traditional methods like core accretion (Pollack et al. 1996) and Class II disk instability (Boss 2011). At wider radii, the most plausible process is likely disk fragmentation at the Class 0/I stage (e.g., Kratter et al. 2010). Nonetheless, PMCs are a potential boon for exoplanet studies; their large separations make them relatively easy to observe, so they could serve as the fully characterized templates against which the more difficult measurements of "traditional" planets are compared.

In particular, PMCs offer an unique window into the process of giant planet assembly and the associated formation of moon systems. Most PMCs have been found in star-forming regions with ages $\lesssim 10 \mathrm{Myr}$, where gas-rich protoplanetary

\footnotetext{
${ }^{10}$ Caltech Joint Center for Planetary Astronomy Fellow.
}

disks are common. Several PMCs exhibit emission lines or possible mid-infrared excesses (Seifahrt et al. 2007; Schmidt et al. 2008; Bowler et al. 2011, 2014; Bailey et al. 2013) that are commonly associated with disks and outflows. Recent Hubble Space Telescope (HST) data confirm that some PMCs have large optical/ultraviolet excess emission, indicative of shocks due to the accretion of disk material (Zhou et al. 2014). Such observations demonstrate that PMCs can host their own circum(sub)stellar disks, composed of material left over from their own formation or accreted from the disk/envelope of their (much more massive) host.

Measurements of the masses, structures, and lifetimes of these disks provide constraints on the assembly timescale for PMCs and the duration of their satellite formation epoch which could be compared with measurements of the complementary freefloating substellar population; (e.g., Liu et al. 2003; Scholz et al. 2006; Andrews et al. 2013). Furthermore, hydrodynamic models of giant planet formation (e.g., Ayliffe \& Bate 2009) make predictions of the disk radii, scale heights, and mass distributions that could be tested with spatially resolved observations, and wide companions also provide context for observations of closein planets with ring systems, such as the recently discovered companion to 1SWASP J140747.93-394542.6 (Mamajek et al. 2012; Kenworthy et al. 2015). However, these disks also pose challenges: if they are oriented at large viewing angles (edgeon; Scholz et al. 2008; Luhman et al. 2007; Looper et al. 2010), the disk material can obscure the PMC and complicate the determination of its mass.

\section{THE FW TAU SYSTEM}

FW Tau is a member of the Taurus-Auriga association, a nearby $(d \sim 140 \mathrm{pc})$, young $(\tau \sim 2 \mathrm{Myr})$ region of ongoing star 
formation. The well-studied primary consists of a close pair ( $\sim 75$ mas, or $11 \mathrm{AU}$, separation) of M5 stars (FW Tau AB) with a total mass of 0.2-0.3 $M_{\odot}$ (Baraffe et al. 1998). FW Tau AB exhibits no spectroscopic evidence of ongoing accretion (Bowler et al. 2014), nor any clear signature of an infrared excess shortward of $24 \mu \mathrm{m}$ (Luhman et al. 2010), indicating that it does not host a significant optically thick disk at orbital radii $<50$ AU. Intriguingly, Andrews \& Williams (2005) noted a $4 \sigma$ detection of the system at $850 \mu \mathrm{m}\left(F_{v}=4.5 \pm 1.1 \mathrm{mJy}\right)$, suggesting that a small amount of cool dust is present within a $\sim 15^{\prime \prime}$ diameter region around the system. Recent Herschel observations by Howard et al. (2013) also find a far-infrared excess within a similar (or larger) beam around FW Tau $\left(F_{v}=30 \pm 4,33 \pm 4\right.$, and $70 \pm 40 \mathrm{mJy}$ at 70,100, and $160 \mu \mathrm{m}$, respectively).

White \& Ghez (2001) first reported an extremely faint candidate (tertiary) companion near FW Tau AB in HST optical images, with anomalously red $i^{\prime} z^{\prime}$ photometry and strong (narrowband) $\mathrm{H} \alpha$ emission. Kraus et al. (2014) subsequently confirmed that this companion (named either FW Tau C or FW Tau $\mathrm{b}$, depending on its poorly understood nature and whether it is best considered as a binary companion or planetary-type companion) and FW Tau AB were co-moving using groundbased adaptive optics imaging, and began a multi-pronged effort to better characterize its properties. If the companion's flux can be attributed to unobscured photospheric emission, the nearinfrared flux suggests a companion mass as low as $10 \mathrm{M}_{\mathrm{Jup}}$, making it another example of a PMC. However, the true mass could be significantly higher or lower if there is an excess from accretion or disk emission (as for many disk-hosting stars and brown dwarfs; Luhman et al. 2010) and/or obscuration from an envelope or edge-on disk (as for a number of other stellar or substellar companions; Stapelfeldt et al. 1998; Scholz et al. 2008; Luhman et al. 2007; Duchêne et al. 2010). Bowler et al. (2014) recently obtained a near-infrared spectrum of FW Tau C that confirms the previous hints of accretion signatures, but found that veiling obscures any photospheric features except broad water absorption bands, which are present for late-M or early-L dwarfs. The spectra therefore remain consistent with either a PMC or a brown dwarf obscured by an edge-on disk.

The tentative detection of submillimeter emission by Andrews \& Williams (2005) from the unresolved FW Tau system hinted that a disk could be present around at least one component, and the high $\mathrm{H} \alpha$ line flux observed from the faint companion by White \& Ghez (2001) strongly suggested that material was accreting onto the companion. In this Letter, we present sensitive, component-resolved $1.3 \mathrm{~mm}$ wavelength observations from the Atacama Large Millimeter Array (ALMA) designed to confirm and localize the putative long-wavelength continuum emission (to FW Tau $\mathrm{AB}$ or to $\mathrm{FW}$ Tau $\mathrm{C}$ ) and to more accurately measure the mass of the dust responsible for it.

\section{ALMA OBSERVATIONS AND DATA REDUCTION}

FW Tau was briefly observed with ALMA in Cycle 1 on 2013 December 2, using 27 available $12 \mathrm{~m}$ antennas in an intermediate configuration (baseline lengths of 17-460 m) and the Band 6 receivers under excellent conditions $(0.6 \mathrm{~mm}$ of precipitable water vapor). The correlator was configured to process four spectral windows in dual polarization, centered at $215,217,230.5$, and $233 \mathrm{GHz}$ (a mean frequency of $224 \mathrm{GHz}$, or $\lambda=1.34 \mathrm{~mm}$ ), each with 128 coarse channels $(15.625 \mathrm{MHz}$ resolution) to maximize continuum sensitivity. Observations cycled between FW Tau and the nearby quasar J051002+180041 on $\sim 7$ minute intervals, with additional visits to J0522-3627

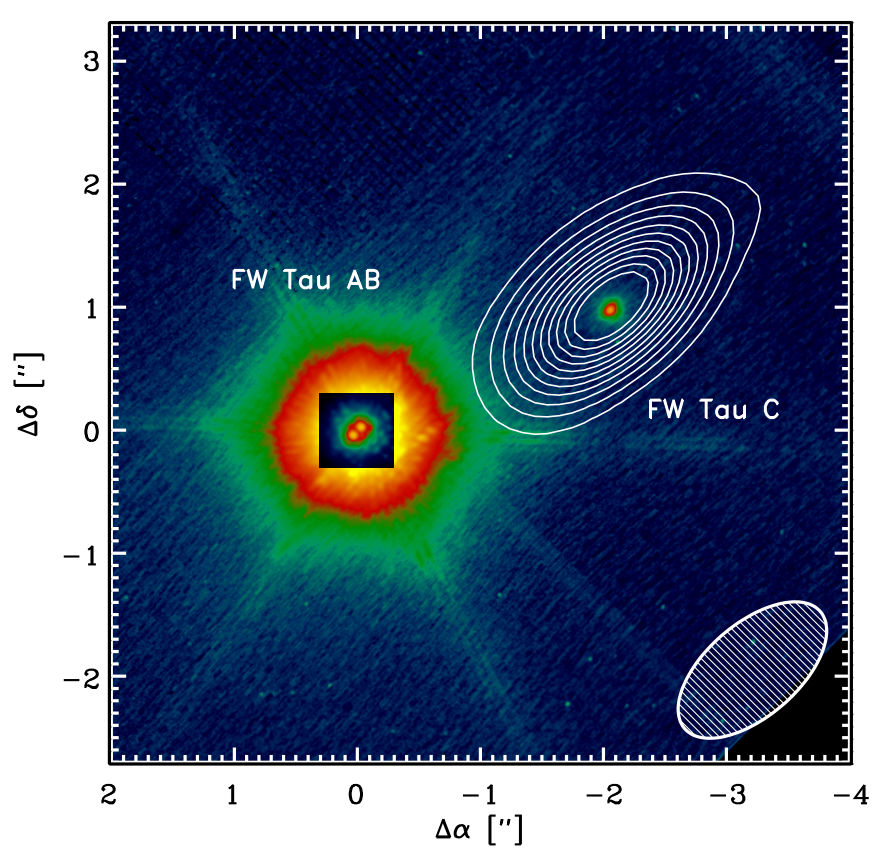

Figure 1. ALMA $1.3 \mathrm{~mm}$ continuum contours ( hown at $5 \sigma \approx 0.14 \mathrm{mJy} \mathrm{beam}^{-1}$ intervals) are overlaid on a near-infrared $\left(K^{\prime}\right)$ image of the FW Tau system taken with the NIRC2 camera on the Keck-II telescope (Kraus et al. 2014). The millimeter-wavelength continuum emission is centered on the faint companion (FW Tau $\mathrm{C}$ or $\mathrm{b}$, depending on its nature as a binary companion or planetary companion), and firmly detected at a peak $\mathrm{S} / \mathrm{N}$ of $\sim 60$. The ALMA synthesized beam dimensions are shown in the lower right corner.

and Ganymede for calibration purposes. The total on-source time for the FW Tau field was 22 minutes.

The raw ALMA visibilities were calibrated and imaged with the CASA software package. After phase correction using the water vapor radiometers, a system temperature correction, and initial flagging (which included the rejection of data from one antenna), the bandpass structure in each spectral window was corrected using observations of J0522-3627. The absolute flux scaling was bootstrapped from observations of Ganymede. Gain variations due to intrinsic changes in the array and atmosphere were determined from the monitoring of J051002+180041 and corrected. The spectrally averaged calibrated visibilities were Fourier inverted (assuming natural weighting), deconvolved with the CLEAN algorithm, and restored with a $1^{\prime \prime} .45 \times 0$ "'.75 $\left(\right.$ P.A. $=131^{\circ}$ ) synthesized beam after a single iteration of phaseonly self-calibration. The rms noise level in the resulting image is $28 \mu \mathrm{Jy}$ beam $^{-1}$.

\section{RESULTS}

Figure 1 shows contours of the ALMA $1.3 \mathrm{~mm}$ continuum emission overlaid on the Keck/NIRC2 $K^{\prime}$ image of the FW Tau components (see Kraus et al. 2014). Continuum emission at $1.3 \mathrm{~mm}$ is firmly detected (peak $\mathrm{S} / \mathrm{N} \approx 60$ ) and centered on the faint companion, located $2^{\prime \prime} .28 \pm 0$ '.05 or $330 \pm 30 \mathrm{AU}$ (P.A. $=$ $\left.296^{\circ} \pm 2^{\circ}\right)$ from the FW Tau AB photocenter $\left(\alpha=04^{\mathrm{h}} 29^{\mathrm{m}} 29^{\mathrm{s}} .71\right.$, $\delta=+26^{\circ} 16^{\prime} 52^{\prime \prime} .82$, based on the ALLWISE astrometry and the proper motion estimated by Kraus et al. 2014). A Gaussian fit to the emission indicates that it is unresolved in the $\sim 200 \times$ $100 \mathrm{AU}$ beam and has an integrated $F_{v}=1.78 \pm 0.03 \mathrm{mJy}$ (with an additional $\sim 10 \%$ systematic uncertainty in the flux scale) as estimated from a Gaussian model of the visibilities.

This millimeter-wave continuum emission is faint enough to be entirely optically thin for any reasonable dust opacity 
(e.g., Beckwith et al. 1990), so the flux measurement can be simply converted into a dust mass estimate. Assuming a characteristic dust temperature of $\sim 10-20 \mathrm{~K}$ and a standard opacity of $2.3 \mathrm{~cm}^{2} \mathrm{~g}^{-1}$ at $1.3 \mathrm{~mm}$, we infer $M_{\text {dust }} \approx 1-2 M_{\oplus}$. This measurement is entirely consistent with the unresolved, marginal detection of $850 \mu \mathrm{m}$ emission $\left(F_{v}=4.5 \pm 1.1 \mathrm{mJy}\right)$ from the FW Tau system by Andrews \& Williams (2005); the corresponding spectrum scales like $F_{v} \propto v^{2.2 \pm 0.6}$, in excellent agreement with the mean value for disks in this wavelength range. The Herschel fluxes from Howard et al. (2013) are also consistent with the far-IR SED that fits our ALMA flux, but do not provide a sufficient constraint on the SED shape to further constrain the dust temperature. The upper limit to the dust emission around the FW Tau AB binary, $F_{v} \leqslant 90 \mu \mathrm{Jy}(3 \sigma)$, is $\sim 30 \times$ lower than typical constraints, and corresponds to a dust mass limit of $\lesssim 0.1 M_{\oplus}$.

The CO $J=3-2$ transition was not detected in the very wide channels (chosen to optimize continuum sensitivity). The $3 \sigma$ upper limit on the peak flux is $\sim 3 \mathrm{mJy}^{\text {beam }}{ }^{-1}$ in a single $20 \mathrm{~km} \mathrm{~s}^{-1}$ wide channel.

\section{DISCUSSION}

The ALMA data clearly show that all of the disk material in the system is associated solely with the faint companion, FW Tau C. Given the dust mass estimated from the observed $1.3 \mathrm{~mm}$ flux $\left(\sim 1-3 M_{\oplus}\right)$ and its complementary signatures of disk accretion (White \& Ghez 2001; Bowler et al. 2014), we have confirmed that the faint companion to FW Tau hosts one of the least massive primordial disks known to date. If that disk proceeds to form its own system of "planetary" or "satellite" companions, they will not achieve sufficient mass to become standard gas or ice giants. However, the mass is well matched to the total mass of compact systems of sub-Earth planets seen around field ultracool dwarfs (e.g., Kepler-42 bcd; Muirhead et al. 2012). It is also interesting to note that the dust mass reservoir still exceeds the total sum of the Galilean satellites (0.066 $M_{\oplus}$; Showman \& Malhotra 1999$)$ by just over an order of magnitude.

Expectations for the disk mass distribution for very lowmass primaries remain uncertain throughout the substellar and planetary mass regime, as there are only a handful of detections and many non-detections (e.g., Scholz et al. 2006; Schaefer et al. 2009; Andrews et al. 2013; Ricci et al. 2014). However, a comparison of the infrared and millimeter fluxes to a population of free-floating young counterparts could still provide context as to the nature of the disk host as either a brown dwarf tertiary companion (FW Tau C) or a PMC (FW Tau b). In Figure 2, we show how the $3.6 \mu \mathrm{m}$ magnitude (converted from the $L^{\prime}$ measurement reported by Kraus et al. 2014) and the new $1.3 \mathrm{~mm}$ flux for the companion compare to the flux versus spectral type relations for disk-bearing stars and brown dwarfs in the Taurus-Auriga region (Luhman et al. 2010; Andrews et al. 2013; Akeson \& Jensen 2014). A shaded band is used for FW Tau C to denote the range of possible spectral types, limited at the upper end ( M5) by the presence of deep water absorption bands in the near-infrared spectrum (Bowler et al. 2014).

At $3.6 \mu \mathrm{m}$, FW Tau C is fainter than nearly all known substellar companions in the region, clearly in the regime of objects with spectral types later than M9 $\left(M<10 M_{\text {Jup }}\right)$, though some edge-on disks also sit well below the median relation. However, comparisons of its millimeter flux with the low-mass Taurus-Auriga population are more ambiguous. Most millimeter-wave surveys for brown dwarf disks were conducted

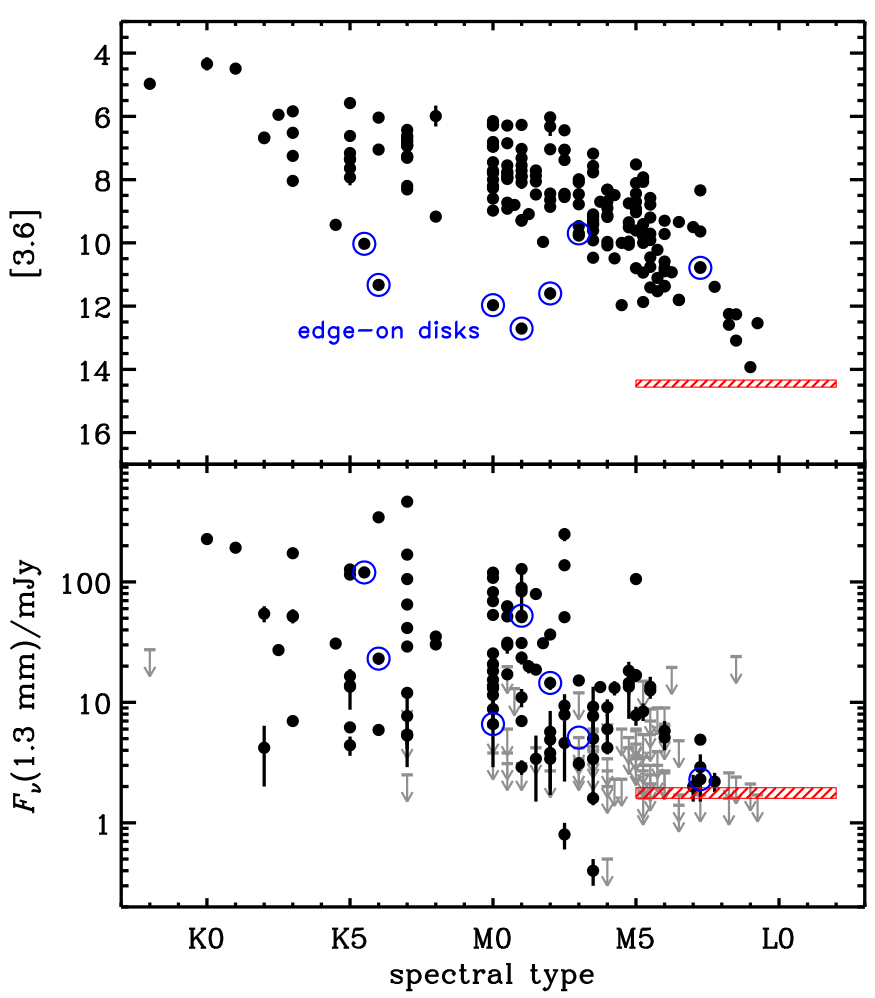

Figure 2. Comparison of FW Tau C (red, shaded bar reflecting the uncertainties) and the population of disk-bearing stars and brown dwarfs in the $\sim 2 \mathrm{Myr}$ old Taurus-Auriga star-forming region, using the empirical scaling of $3.6 \mu \mathrm{m}$ magnitudes and $1.3 \mathrm{~mm}$ fluxes ( $3 \sigma$ upper limits are marked as gray arrows) with spectral type. The Taurus-Auriga data were collected as described by Andrews et al. (2013), Akeson \& Jensen (2014), and Luhman et al. (2010); the FW Tau C measurements were converted from the Kraus et al. (2014) $L^{\prime}$ photometry (using their conversion relation between the bands) and the ALMA data presented here. We use blue circles to denote the six stars in this sample that are either known or strongly suspected to have edge-on disk orientations, either from direct high-resolution imaging (HK Tau B, HV Tau C, 2MASS J04202144+2813491, and 2MASS J04381486+2611399; Stapelfeldt et al. 1998; Luhman et al. 2007, 2010; Duchêne et al. 2010) or a combination of an anomalously low $L_{*}$ (a $>2 \sigma$ deviation from the mean luminosity at that spectral type), high $A_{V}$, or unusual optical/near-infrared SED morphology (IRAS 04260+2642, IRAS 04301+2608, and ITG 33A; Andrews et al. 2013).

with facilities that are substantially less sensitive than ALMA. The best available upper limits of those surveys approach the $1.3 \mathrm{~mm}$ flux detected here for FW Tau C, so there are at least some brown dwarf disks with masses that could be quite a bit lower. The disk mass measured here is consistent with the range of possible values for isolated Taurus-Auriga brown dwarfs with spectral types later than $\sim \mathrm{M} 5$, assuming it has a similar age and formed in a similar process.

A more appropriate comparison would consider only companions, rather than the full population of isolated objects. Even a very low-mass companion might be able to retain a substantial disk via Bondi-Hoyle accretion from the disk or envelope of the primary, but free-floating objects do not have such a reservoir to build or maintain a comparable disk mass. Unfortunately, the number of substellar companions in nearby star-forming regions is quite low, and the number with component-resolved millimeter measurements is even lower, so such a comparison is not yet feasible.

Trends observed for more massive stars might also apply, but it is unclear which processes are dominant in this specific case. A clear trend is seen in older regions for less massive free-floating objects to retain their disks longer (Carpenter et al. 
2006), perhaps even for several tens of Myr among brown dwarfs (Riaz \& Gizis 2008; Reiners 2009). Observations of (stellar) binaries in Taurus suggest that primaries usually dominate the disk mass budget (Harris et al. 2012), though perhaps not out of proportion to the observed scaling of disk mass with primary mass (Akeson \& Jensen 2014), and the disks ultimately seem to have similar lifetimes (Prato \& Simon 1997; Daemgen et al. 2012). Furthermore, close binaries (such as the FW Tau AB pair, with $\rho \sim 15 \mathrm{AU}$ ) appear to have sharply reduced disk frequencies even at early ages (White \& Ghez 2001; Cieza et al. 2009; Duchêne 2010; Kraus et al. 2012), so it might not be surprising that the primary has cleared its disk quickly. The relative rate of disk dispersal is therefore an ambiguous feature in the classification of this system.

In the meantime, the most unambiguous way to determine the mass and geometry of FW Tau $\mathrm{C}$ will be to spectroscopically detect photospheric absorption features, either at higher spectral resolution or in the $\sim 1 \mu \mathrm{m}$ regime where veiling is minimized. Disk mass determinations for more securely identified PMCs also could shed light on the distinction between companions formed via binary processes or planetary processes. In any case, the ALMA data presented here signals that we are entering a new regime of sensitivity that will ultimately enable the characterization of fundamental circum(sub-)stellar and circumplanetary disk properties.

The authors thank Gaspard Duchêne for an insightful discussion of the nature of the FW Tau companion, and the referee for a helpful critique of this Letter. This Letter makes use of the following ALMA data: ADS/JAO.ALMA\#2012.1.00989.S. ALMA is a partnership of ESO (representing its member states), NSF (USA) and NINS (Japan), together with NRC (Canada) and NSC and ASIAA (Taiwan), in cooperation with the Republic of Chile. The Joint ALMA Observatory is operated by ESO, AUI/ NRAO and NAOJ. The NRAO is a facility of the NSF operated under cooperative agreement by Associated Universities, Inc.

\section{REFERENCES}

Akeson, R. L., \& Jensen, E. L. N. 2014, ApJ, 784, 62

Andrews, S. M., Rosenfeld, K. A., Kraus, A. L., \& Wilner, D. J. 2013, ApJ, 771,129

Andrews, S. M., \& Williams, J. P. 2005, ApJ, 631, 1134

Ayliffe, B. A., \& Bate, M. R. 2009, MNRAS, 397, 657

Bailey, V., Hinz, P. M., Currie, T., et al. 2013, ApJ, 767, 31
Bailey, V., Meshkat, T., Reiter, M., et al. 2014, ApJL, 780, L4

Baraffe, I., Chabrier, G., Allard, F., \& Hauschildt, P. H. 1998, A\&A, 337, 403

Beckwith, S. V. W., Sargent, A. I., Chini, R. S., \& Guesten, R. 1990, AJ, 99, 924

Boss, A. P. 2011, ApJ, 731, 74

Bowler, B. P., Liu, M. C., Kraus, A. L., \& Mann, A. W. 2014, ApJ, 784, 65

Bowler, B. P., Liu, M. C., Kraus, A. L., Mann, A. W., \& Ireland, M. J. 2011, ApJ, 743,148

Carpenter, J. M., Mamajek, E. E., Hillenbrand, L. A., \& Meyer, M. R. 2006, ApJL, 651, L49

Cieza, L. A., Padgett, D. L., Allen, L. E., et al. 2009, ApJL, 696, L84

Daemgen, S., Correia, S., \& Petr-Gotzens, M. G. 2012, A\&A, 540, A46

Duchêne, G. 2010, ApJL, 709, L114

Duchêne, G., McCabe, C., Pinte, C., et al. 2010, ApJ, 712, 112

Harris, R. J., Andrews, S. M., Wilner, D. J., \& Kraus, A. L. 2012, ApJ, 751, 115

Howard, C. D., Sandell, G., Vacca, W. D., et al. 2013, ApJ, 776, 21

Ireland, M. J., Kraus, A., Martinache, F., Law, N., \& Hillenbrand, L. A. 2011, ApJ, 726, 113

Kenworthy, M. A., Lacour, S., Kraus, A., et al. 2015, MNRAS, 446, 411

Kratter, K. M., Murray-Clay, R. A., \& Youdin, A. N. 2010, ApJ, 710, 1375

Kraus, A. L., \& Ireland, M. J. 2012, ApJ, 745, 5

Kraus, A. L., Ireland, M. J., Cieza, L. A., et al. 2014, ApJ, 781, 20

Kraus, A. L., Ireland, M. J., Hillenbrand, L. A., \& Martinache, F. 2012, ApJ, 745,19

Kuzuhara, M., Tamura, M., Kudo, T., et al. 2013, ApJ, 774, 11

Lafrenière, D., Jayawardhana, R., \& van Kerkwijk, M. H. 2008, ApJL, 689, L153

Lagrange, A., Gratadour, D., Chauvin, G., et al. 2009, A\&A, 493, L21

Liu, M. C., Najita, J., \& Tokunaga, A. T. 2003, ApJ, 585, 372

Looper, D. L., Mohanty, S., Bochanski, J. J., et al. 2010, ApJ, 714, 45

Luhman, K. L., Adame, L., D’Alessio, P., et al. 2007, ApJ, 666, 1219

Luhman, K. L., Allen, P. R., Espaillat, C., Hartmann, L., \& Calvet, N. 2010, ApJS, 186, 111

Mamajek, E. E., Quillen, A. C., Pecaut, M. J., et al. 2012, AJ, 143, 72

Marois, C., Macintosh, B., Barman, T., et al. 2008, Sci, 322, 1348

Muirhead, P. S., Johnson, J. A., Apps, K., et al. 2012, ApJ, 747, 144

Neuhäuser, R., Guenther, E. W., Wuchterl, G., et al. 2005, A\&A, 435, L13

Pollack, J. B., Hubickyj, O., Bodenheimer, P., et al. 1996, Icar, 124, 62

Prato, L., \& Simon, M. 1997, ApJ, 474, 455

Rameau, J., Chauvin, G., Lagrange, A.-M., et al. 2013, ApJL, 772, L15

Reiners, A. 2009, ApJL, 702, L119

Riaz, B., \& Gizis, J. E. 2008, ApJ, 681, 1584

Ricci, L., Testi, L., Natta, A., et al. 2014, ApJ, 791, 20

Schaefer, G. H., Dutrey, A., Guilloteau, S., Simon, M., \& White, R. J. 2009, ApJ, 701,698

Schmidt, T. O. B., Neuhäuser, R., Seifahrt, A., et al. 2008, A\&A, 491, 311

Scholz, A., Jayawardhana, R., \& Wood, K. 2006, ApJ, 645, 1498

Scholz, A., Jayawardhana, R., Wood, K., et al. 2008, ApJL, 681, L29

Seifahrt, A., Neuhäuser, R., \& Hauschildt, P. H. 2007, A\&A, 463, 309

Showman, A. P., \& Malhotra, R. 1999, Sci, 296, 77

Stapelfeldt, K. R., Krist, J. E., Menard, F., et al. 1998, ApJL, 502, L65

White, R. J., \& Ghez, A. M. 2001, ApJ, 556, 265

Zhou, Y., Herczeg, G. J., Kraus, A. L., Metchev, S., \& Cruz, K. L. 2014, ApJL, 783, L17 